\title{
Photopolymerization of Styrene with Azo-Containing Polydimethylsiloxane as Photoinitiator
}

\author{
Te-Chuan Chang, ${ }^{\dagger}$ Hon-Bin ChEN, ${ }^{*}$ and Kuo-Hui Wu \\ Department of Applied Chemistry, Chung Cheng Institute of Technology, \\ Taoyuan, Taiwan 33509, Republic of China \\ * Chemical Systems Research Division, Chung Shan Institute of Science and \\ Technology, Taoyuan, Taiwan 32526, Republic of China
}

(Received October 25, 1996)

\begin{abstract}
Azo-containing polydimethylsiloxane (PDMS-ACP), prepared by polycondensation reaction of bis(hydroxybutyl) polydimethylsiloxane (PDMS) with 4,4'-azobis-4-cyanopentanoyl chloride (ACPC), was used as the photoinitiator for the polymerization of styrene (St) in bulk. Polymerization of styrene with azobisisobutyronitrile (AIBN) as photoinitiator was conducted for comparision. The propagation and termination rate constants $\left(k_{\mathrm{p}}\right.$ and $\left.k_{\mathrm{t}}\right)$, and the fractions $\beta$ of primary radicals entering into termination were calculated. The values of $k_{\mathrm{p}}$ and $k_{\mathrm{t}}$ in St/PDMS-ACP system were in agreement with that in St/AIBN system. However, the photoinitiator efficiency $\Phi$ of PDMS-ACP was found to be smaller than that of AIBN. The ratio $\sigma k_{\mathrm{t}} / k_{\mathrm{i}}$ of the rate constants for chain termination and chain initiation by primary radicals were determined.

KEY WORDS Macroinitiator/Rate Constant / Photopolymerization / Styrene /
\end{abstract}

Block copolymers composed of a condensation polymer segments (e.g., polyether, polyester, polyamide, polyurethane, and polysiloxane) with an additional polymer segments (e.g., selected from various types of vinyl polymers) are highly attractive polymers. This type of block copolymers has potential to exhibit a wide variety of new physical and chemical characteristics depending on the combination of the natures of the polymer segments chosen. $^{1,2}$

Polydimethylsiloxane (PDMS) repeating - $\mathrm{OSi}\left(\mathrm{CH}_{3}\right)_{2}-$ unit is indeed endowed with unusual properties such as high dynamic flexibility, low entropy of dilution, higher oxidative stability, excellent thermal stability, high lubricity, and low glass transition temperature. ${ }^{3}$ On the other hand, polystyrene (PSt) has been widely used for many years as a general-purpose thermoplastic in the form of molding, electrical insulation, packaging, etc. Therefore, there has been considerable effort to incorporate PDMS into styrene block polymers by anionic polymerization method, ${ }^{4}$ condensation polymerization, ${ }^{5}$ and free-radical routes. ${ }^{6}$

Heitz et al. prepared the azo-containing macroinitiators and the copolymers by use of this macroinitiator. ${ }^{7}$ Thereafter, azo-containing macroinitiators for radical polymerization have been studied. ${ }^{8}$ We reported recently the activation energy of thermal decomposition of the azo-containing polydimethylsiloxane (PDMSACP) macroinitiator. ${ }^{9}$ Moreover, the apparent activation energy in thermal degradation of the PDMS- $b$ PMMA block copolymers was studied by thermogravimetric analysis. ${ }^{10}$ Furthermore, the kinetic parameters of photopolymerization for methyl methacrylate (MMA) with PDMS-ACP as initiator were also evaluated. ${ }^{11}$ However, the kinetic parameters of photopolymerization for styrene with PDMS-ACP as initiator are not evaluated. The purpose of this work is to evaluate the propagation rate constant $k_{\mathrm{p}}$ and termination rate constant $k_{\mathrm{t}}$ of styrene with PDMS-ACP as photoinitiator

$\dagger$ To whom correspondence should be addressed.

442 in bulk. The initiator efficiency $\theta$, the fraction $\beta$ of primary radicals entering into termination and the ratio $\sigma k_{\mathrm{t}} / k_{\mathrm{i}}$ of the rate constants for chain termination and chain initiation by primary radicals are also investigated.

\section{EXPERIMENTAL}

\section{Materials}

4,4'-Azobis-4-cyanopentanoic acid (ACPA; Aldrich), thionyl chloride (Aldrich), triphenylformazan (Tokyo Kasei Co.), and the solvents (reagent grade, Merck) were used without purification. Octamethylcyclotetrasiloxane $\left(\mathrm{D}_{4}\right.$; polymerization grade, Hüls-Petrarch) was vacuum distilled over $\mathrm{CaH}_{2}$ prior to its use. 1,3-Bis(4hydroxybutyl)tetramethyldisiloxane (polymerization grade, Hüls-Petrarch) was used as received. The monomer styrene (Janssen) was purified by distillation before use. Azobisisobutyronitrile (AIBN; BDH) was recrystallized from ethanol before use. Macroinitiator of the PDMS-ACP was prepared as described previously (Scheme 1). ${ }^{11}$

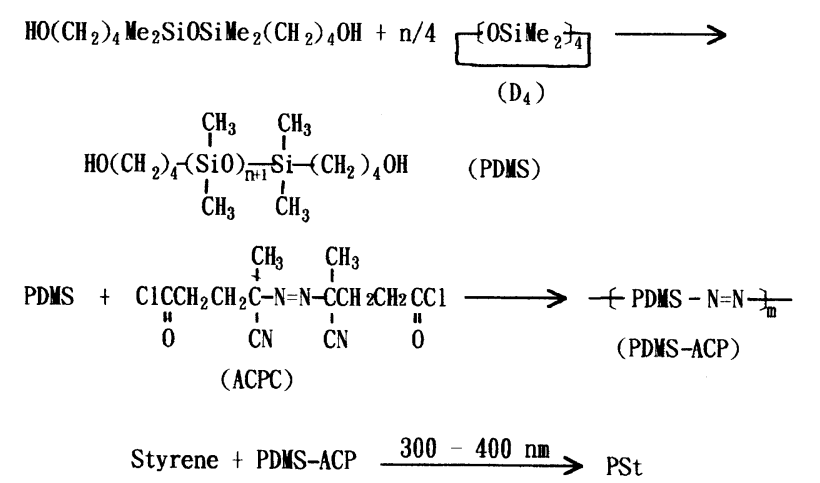

Scheme 1.

\section{Photopolymerization}

The reaction mixture of styrene and the initiator (PDMS-ACP or AIBN) was placed into a dilatometer $(25 \mathrm{~mL})$ which was degassed by the typical freeze-thaw 
technique and sealed under vacuum $\left(10^{-4} \mathrm{mmHg}\right)$. The Pyrex dilatometer was kept at $30 \pm 0.05^{\circ} \mathrm{C}$ in a water bath. The light source was a high-pressure mercury lamp (Osram $200 \mathrm{~W}$ ) equipped with a filter to pass $300-400 \mathrm{~nm}$.

Rate of polymerization $R_{\mathrm{p}}$ was determined by measuring the variation of the solution meniscus in the capillary tube of the dilatometer as a function of duration of reaction. The rate of initiation $R_{\mathrm{i}}$ was determined according to the inhibition method, and the scavenger 1,3,5-triphenylverdazyl (TPV) was prepared from triphenylformazan (Tokyo Kasei Co.). ${ }^{12}$ The average lifetime of radicals $(\tau)$ was determined with a rotting sector as described before. ${ }^{11,13}$

\section{Characterization}

The number-average molar molecular weight $\left(\bar{M}_{n}=\right.$ 3000 ) of the PDMS was calculated from the absorption of the butyl group for PDMS relative to $2.0 \mathrm{ppm}$ (internal standard; $\mathrm{CH}_{3} \mathrm{CN}$ ) in ${ }^{1} \mathrm{H}$ NMR spectrum; the molecular weight dispersity of the PDMS $(D=2.0)$ was calculated based on polystyrene standards from gel permeation chromatography (GPC; Waters).

The azo group content in PDMS-ACP was $2.9 \times$ $10^{-4} \mathrm{molg}^{-1}$ as determined from the intensity in the UV spectrum at $348 \mathrm{~nm}$ based on AIBN $\left(\varepsilon_{348}=14.7\right.$ $\left.\mathrm{K} \mathrm{mol}^{-1} \mathrm{~cm}^{-1}\right) .{ }^{9}$ GPC (THF): $\left(\bar{M}_{n}\right)=7300, D=1.8$.

The precipitated polymer was filtered and washed with methanol, and purified by three successive precipitation from chloroform solutions by adding methanol. Numberaverage molecular weight $\left(\bar{M}_{n}\right)$ and molecular weight dispersity $(D)$ of polymers were measured by GPC, with columns (Shodex KF $801+802+803+804)$, and tetrahydrofuran as eluent $\left(1 \mathrm{~mL} \mathrm{~min}^{-1}\right)$.

\section{RESULTS AND DISCUSSION}

The photolysis of PDMS-ACP may initiate the polymerization of styrene. The rate of polymerization $R_{\mathrm{p}}$ of styrene with PDMS-ACP as initiator is in agreement with that with AIBN as initiator (Table I). Moreover, the number-average molecular weights of polymers prepared with the PDMS-ACP as initiator is found to be similar with those obtained from AIBN as initiator

Table I. The rates of photopolymerization $\left(R_{\mathrm{p}}\right)$, initiator efficiency $(\Phi)$, the fraction of primary radical terminating the chain $(\beta)$, thenumber-average molar mass $\left(\bar{M}_{n}\right)$ of the polymers, and the molecular weight distribution $(D)$ obtained for the photopolymerization of styrene in bulk with PDMS-ACP and AIBN as initiator

\begin{tabular}{|c|c|c|c|c|c|}
\hline \multirow{2}{*}{ Initiator } & \multirow{2}{*}{$\frac{[\mathrm{I}]^{\mathrm{a}}\left(\times 10^{2}\right)}{\mathrm{mol} \mathrm{L}^{-1}}$} & \multirow{2}{*}{$\frac{R_{\mathrm{p}}\left(\times 10^{5}\right)}{\mathrm{L} \mathrm{mol}^{-1} \mathrm{~s}^{-1}}$} & \multirow{2}{*}{$\Phi$} & \multirow{2}{*}{$\beta$} & \multirow{2}{*}{$\begin{array}{c}\bar{M}_{n}(D) \\
\left(\times 10^{3} \mathrm{~g}\right)\end{array}$} \\
\hline & & & & & \\
\hline \multirow[t]{4}{*}{ PDMS-ACP } & 0.2 & 2.6 & 0.61 & 0.18 & $106.8(1.7)$ \\
\hline & 0.5 & 3.4 & 0.43 & 0.21 & $76.8(1.8)$ \\
\hline & 1.0 & 4.4 & 0.37 & 0.24 & $42.1(1.7)$ \\
\hline & 1.5 & 4.9 & 0.32 & 0.25 & $34.2(1.9)$ \\
\hline \multirow[t]{4}{*}{ AIBN } & 0.2 & 2.0 & 0.85 & 0.06 & $100.3(1.8)$ \\
\hline & 0.5 & 3.3 & 0.91 & 0.09 & $56.9(1.8)$ \\
\hline & 1.0 & 4.2 & 0.73 & 0.11 & $37.1(2.0)$ \\
\hline & 1.5 & 4.9 & 0.67 & 0.13 & $30.1(2.1)$ \\
\hline
\end{tabular}

${ }^{a}$ Concentration of azo groups.
(Table I).

The value of $R_{\mathrm{p}}$ increases in bulk polymerization with an increase in the concentration of initiators. Plots of $\log R_{\mathrm{p}}$ vs. $\log [\mathrm{I}]$ give straight lines with slopes of 0.27 for St/PDMS-ACP system and 0.44 for St/AIBN system, respectively. [I] is the mean concentration of azo groups that is also equal to the mean concentration of the monofunctional group initiator (AIBN) in the system. The reaction order 0.27 with respect to initiator PDMSACP was found to be much less than 0.5. This result may be due to decrease in initiator efficiency $\Phi$. Alternatively, the termination process may alter from the normal bimolecular termination between two chain radicals $(\mathbf{P} \cdot)$ to termination by reaction of a chain radical with a primary radical $(\mathrm{R} \cdot),{ }^{14}$ particularly in St/PDMSACP system.

$$
\begin{aligned}
& \mathrm{P} \cdot+\mathrm{P} \cdot \stackrel{k_{\mathrm{t}}}{\longrightarrow} \text { polymer products } \\
& \mathrm{P} \cdot+\mathrm{R} \cdot \stackrel{\sigma k_{\mathrm{t}}}{\longrightarrow} \text { polymer products }
\end{aligned}
$$

where $k_{\mathrm{t}}$ and $\sigma k_{\mathrm{t}}$ are the rate constants for two chain radicals termination and for a primary radical termination, respectively. Here $\sigma$ is a constant.

The relationship between $R_{\mathrm{p}},[\mathrm{M}]$ and [I] is given by ${ }^{15}$ :

$$
[\mathrm{M}] / R_{\mathrm{p}}=\frac{\lambda}{[\mathrm{I}]^{0.5}}+\frac{\mu}{[\mathrm{M}]}
$$

where $\lambda$ is the value of $\left(k_{\mathrm{t}} / 2 k_{\mathrm{d}}\right)^{0.5} / k_{\mathrm{p}}, \mu$ is the value of $\sigma k_{\mathrm{t}} / k_{\mathrm{i}} k_{\mathrm{p}}$, and [M] is the monomer concentration. A plot of $[\mathrm{M}] / R_{\mathrm{p}}$ vs. $1 /[\mathrm{I}]^{0.5}$ at constant monomer concentration gives straight lines (Figure 1) for PDMS-ACP and AIBN, as predicted by eq 3 . Accordingly, it is considered that initiators also act as terminators in the photopolymerization of styrene. The values of $\lambda$ and $\mu$ are obtained from the slopes and the intercepts of the straight lines, respectively. The resulting values are listed in

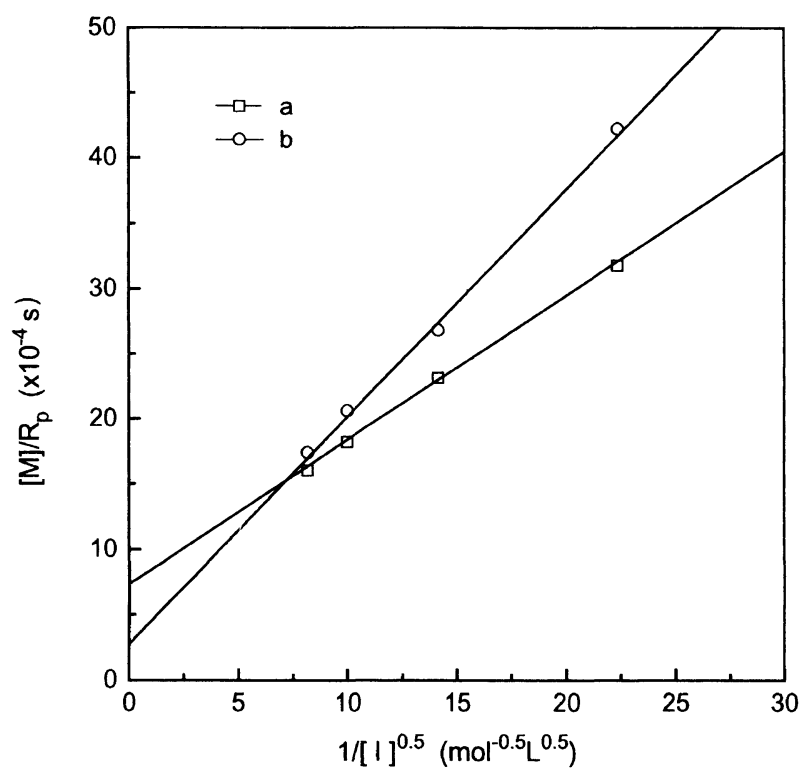

Figure 1. Plots of equation (3) showing the dependence of the rate of polymerization on initiator concentration: (a) PDMS-ACP; (b) AIBN. 
Table II. Kinetic parameters of photopolymerization of styrene in bulk

\begin{tabular}{lrr}
\multicolumn{1}{c}{ Parameters } & \multicolumn{2}{c}{ Initiator $^{\mathrm{a}}$} \\
\cline { 2 - 3 } & PDMS-ACP & AIBN \\
\hline$R_{\mathrm{p}} / \times 10^{-5} \mathrm{M} \mathrm{s}^{-1}$ & 4.4 & 4.2 \\
$R_{\mathrm{i}} / \times 10^{-8} \mathrm{M} \mathrm{s}^{-1}$ & 9.2 & 7.9 \\
$\tau / \mathrm{s}$ & 1.0 & 1.2 \\
$k_{\mathrm{p}} / \mathrm{M}^{-1} \mathrm{~s}^{-1}$ & 55.6 & 53.4 \\
$k_{\mathrm{t}} / \times 10^{7} \mathrm{M}^{-1} \mathrm{~s}^{-1}$ & 1.1 & 0.9 \\
$\lambda / \times 10^{3} \mathrm{M}^{0.5} \mathrm{~s}$ & 11.0 & 17.4 \\
$\mu / \times 10^{5} \mathrm{M} \mathrm{s}^{-1}$ & 5.8 & 2.3 \\
$k_{\mathrm{d}} / \times 10^{-6} \mathrm{~s}^{-1}$ & 12.4 & 5.4 \\
$\sigma k_{\mathrm{t}} k_{\mathrm{i}}^{-1} / \times 10^{7}$ & 3.2 & 1.2 \\
\hline
\end{tabular}

${ }^{a}$ Concentration of azo group $=0.01 \mathrm{~mol} \mathrm{~L}^{-1}$.

Table II. It is found that the value of $\mu$ in st/PDMSACP system is larger than that in St/AIBN system. The result implies that fraction $\beta$ of primary radical termination in macroinitiator PDMS-ACP is larger than that in AIBN initiator.

The fraction $\beta$ of primary radicals entering into termination was calculated by ${ }^{15}$ :

$$
\beta=\sigma R_{\mathrm{t}} / R_{\mathrm{d}}=\frac{\Phi^{0.5}}{1+\left(\lambda[\mathrm{M}] / \mu[\mathrm{I}]^{0.5}\right)}
$$

where $\sigma R_{\mathrm{t}}$ and $R_{\mathrm{d}}$ are the rate of termination by primary radicals and the rate of initiator decomposition, respectively. The value of initiator efficiency $\Phi$ can be obtained from the known values of $\lambda, R_{\mathrm{p}},[\mathrm{I}]^{0.5}$ and $[\mathrm{M}]$ according to the following equation ${ }^{15}$ :

$$
\Phi^{0.5}=\lambda R_{\mathrm{p}} /[\mathrm{I}]^{0.5}[\mathrm{M}]
$$

The values of $\Phi$ and $\beta$ are listed in Table I for polymerization of styrene. The data indicate that the values of $\Phi$ in both polymerizations decrease with increase in concentration of initiator, whereas the values of $\beta$ increase. Moreover, the value of $\Phi$ for the polymerization of styrene with PDMS-ACP as initiator is smaller than that with AIBN as initiator, whereas the value of $\beta$ in the St/PDMS-ACP system is larger than that in the $\mathrm{St} /$ AIBN system.

1,3,5-Triphenylverdazyl (TPV) was used as the scavenger, which traps a primary radical in the polymerization system [eq 6], to determine the rate of initiation $\left(R_{\mathrm{i}}\right)$. The inhibiting effect of TPV on photopolymerization in the St/PDMS-ACP system is shown in Figure 2. Generally, inhibition mechanism should be same regardless of concentration TPV, giving same slopes. However, the slope at high concentration of TPV is different from that at low concentration in St/PDMS-ACP system. The result is also observed at $\mathrm{St} / \mathrm{AIBN}$ system. It is revealed that the products of TPV inhibition with primary radicals show the secondary inhibiting action. ${ }^{16}$ The correlation between the concentration of scavenger [TPV] and the duration of inhibition in the St/PDMSACP system is shown in Figure 3. The rate of initiation $R_{\mathrm{i}}$ was calculated from the slope of Figure 3 according to eq 7. $R_{\mathrm{i}}$ of styrene with PDMS-ACP as initiator $\left(9.2 \times 10^{-8} \mathrm{~mol} \mathrm{~L}^{-1} \mathrm{~s}^{-1}\right)$ is in agreement with that with AIBN as initiator $\left(7.9 \times 10^{-8} \mathrm{~mol} \mathrm{~L}^{-1} \mathrm{~s}^{-1}\right)$. Moreover, the value of $k_{\mathrm{p}}^{2} / k_{\mathrm{t}}$ is derived from eq 8 .

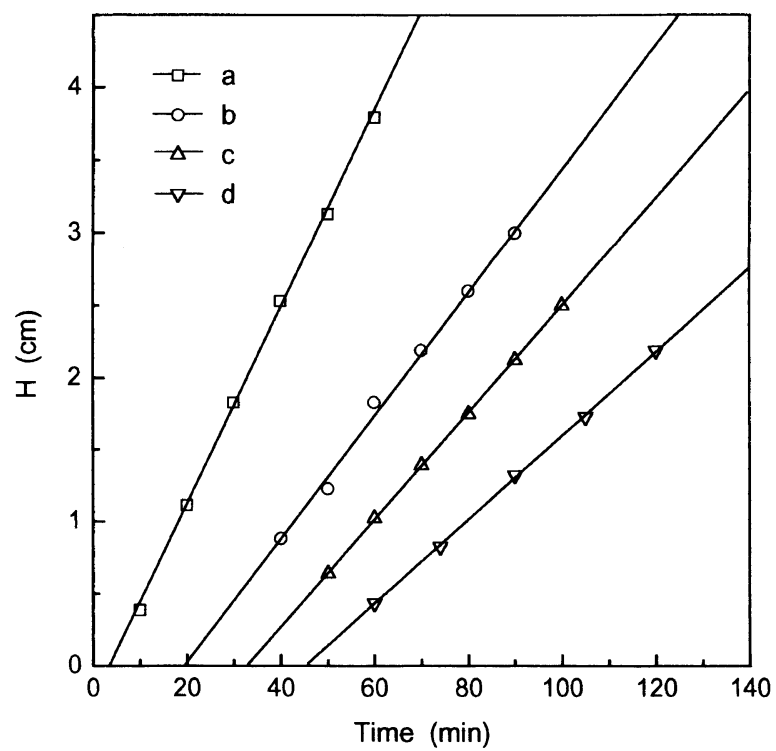

Figure 2. The TPV inhibition effect on the photopolymerization of styrene in bulk initiated by PDMS-ACF (concentration of azo group = $0.01 \mathrm{~mol} \mathrm{~L}^{-1}$ ); [TPV] (in $\mathrm{mmol} \mathrm{L}^{-1}$ ): (a) 0 ; (b) 13 ; (c) 19 ; (d) 26

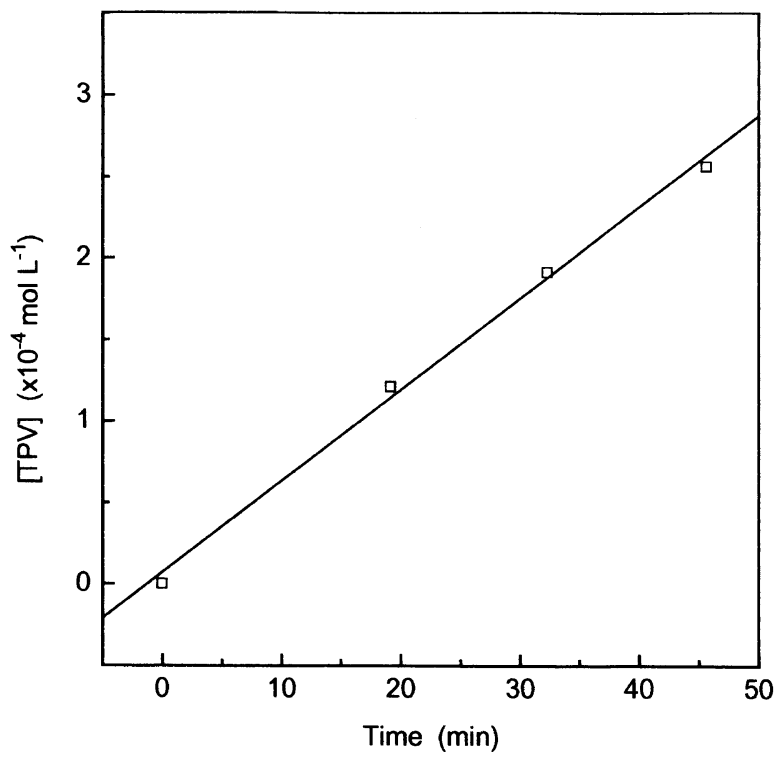

Figure 3. Plots of $[\mathrm{TPV}] v s$. induction time in polymerization of styrene in bulk with PDMS-ACP (concentration of azo group= $0.01 \mathrm{~mol} \mathrm{~L}^{-1}$ ).
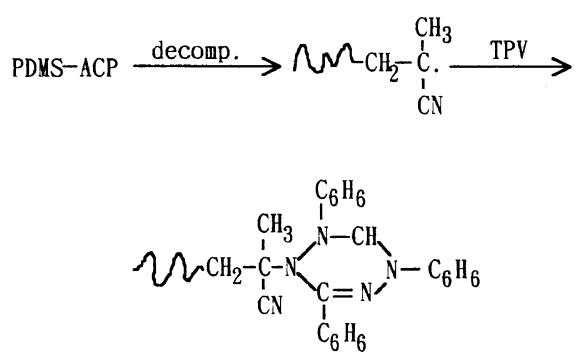

$$
\begin{gathered}
R_{\mathrm{i}}=\frac{[\mathrm{TPV}]_{0}}{\Delta t}=\frac{\text { Initial concentration of scavenger }}{\text { Time of inhibition period }} \\
\frac{k_{\mathrm{p}}^{2}}{k_{\mathrm{t}}}=\frac{R_{\mathrm{p}}^{2}}{R_{\mathrm{i}}[\mathrm{M}]^{2}}
\end{gathered}
$$


The average lifetime $\tau$ of the chain radicals can be determined by the rotating sector method. The data are listed in Table II. The value of $\tau$ in St/PDMS-ACP system $(1.0 \mathrm{~s})$ is close to St/AIBN system $(1.2 \mathrm{~s})$. In addition, the value of $k_{\mathrm{p}} / k_{\mathrm{t}}$ is derived from eq 9 .

$$
\frac{k_{\mathrm{p}}}{k_{\mathrm{t}}}=\frac{R_{\mathrm{p}} \tau}{[\mathrm{M}]}
$$

The values of the rate constant of polymerization $k_{\mathrm{p}}$ and of termination $k_{\mathrm{t}}$ are then calculated from eq 8 and 9 . The rate of constants $k_{\mathrm{p}}$ and $k_{\mathrm{t}}$ are 55.6 and $1.1 \times 10^{7}$ $\mathrm{L} \mathrm{mol}^{-1} \mathrm{~s}^{-1}$ in the St/PDMS-ACP system, and 53.4 and $9.0 \times 10^{6} \mathrm{~L} \mathrm{~mol}^{-1} \mathrm{~s}^{-1}$ in the St/AIBN system, respectively. These values of $k_{\mathrm{p}}$ and $k_{\mathrm{t}}$ agree with the literature values. ${ }^{17}$ On the other hand, the number-average molecular weight $\left(\bar{M}_{n}\right)$ of the polymers prepared with PDMSACP as initiator are found to be similar with those with AIBN as initiator (Table I). This observation suggests that the propagation radicals are less effective by the PDMS segments in the St/PDMS-ACP system.

The decomposition rate constant $k_{\mathrm{d}}$ for initiator can be calculated by:

$$
\begin{aligned}
& \lambda=\left(k_{\mathrm{t}} / 2 k_{\mathrm{d}}\right)^{0.5} / k_{\mathrm{p}} \\
& k_{\mathrm{d}}=k_{\mathrm{t}} / 2\left(\lambda k_{\mathrm{p}}\right)^{2}
\end{aligned}
$$

The value of $k_{\mathrm{d}}$ for PDMS-ACP $\left(12.4 \times 10^{-6} \mathrm{~s}^{-1}\right)$ is notably greater than that for AIBN $\left(5.4 \times 10^{-6} \mathrm{~s}^{-1}\right)$. Moreover, the value of $k_{\mathrm{d}}$ for PDMS-ACP initiator in styrene is in agreement with that in methyl methacrylate $\left(15.5 \times 10^{-6} \mathrm{~s}^{-1}\right) .{ }^{11} \mathrm{On}$ the other hand, the ratio of the rate constants of termination and initiation by primary radicals $\sigma k_{\mathrm{t}} / k_{\mathrm{i}}$ can be obtained from the known values of $\mu$ and $k_{\mathrm{p}}$ according to the following equation:

$$
\begin{aligned}
& \mu=\sigma k_{\mathrm{t}} / k_{\mathrm{i}} k_{\mathrm{p}} \\
& \sigma k_{\mathrm{t}} / k_{\mathrm{i}}=\mu k_{\mathrm{p}}
\end{aligned}
$$

The values of $\sigma k_{\mathrm{t}} / k_{\mathrm{i}}$ are $3.2 \times 10^{7}$ and $1.2 \times 10^{7}$ for PDMS-ACP and AIBN, respectively. The results reveal that the rate of primary radical termination in PDMSACP macroazoinitiator is greater than that in AIBN.
Taking into account that PDMS-ACP macroinitiator gives a small initiator efficiency $\Phi$, and a greater fraction $\beta$ of primary radicals termination, it may be concluded that the increased size of the primary radicals reduces their diffusion apart, thus increases the chance of recombination. ${ }^{18}$

Acknowledgment. We thank the National Science Council of the Republic of China for financial support (NSC 86-2113-M-014-003).

\section{REFERENCES}

1. R. P. Kambour, H. J. Klopfer, and S. A. Smith, J. Appl. Polym Sci., 26, 847 (1986).

2. A. Ueda and S. Nagai, J. Polym. Sci., Polym. Chem. Ed., 24, 405 (1986).

3. S. J. Clarson and J. A. Semlyen, "Siloxane Polymers," Ellis Horwood-PTR, Prentice Hall, New York, N.Y., 1993, p. 72.

4. S. J. Clarson, J. O. Stuart, C. E. Selby, A. Sabata, S. D. Smith, and A. Ashraf, Macromolecules, 28, 674 (1995).

5. P. Chaumont, G. Beinert, J. Herz, and P. Rempp, Eur. Polym. J., 15, 459 (1979).

6. H. Inoue, A. Matsumoto, K. Matsukawa, A. Ueda, and S. Nagai, J. Appl. Polym. Sci., 40, 1917 (1990).

7. R. Walz, B. Bomer, and W. Heitz, Makromol. Chem., 178, 2527 (1977).

8. H. Kinoshita, N. Tanaka, and T. Araki, Makromol. Chem., 194, 2335 (1993).

9. T. C. Chang, H. B. Chen, Y. C. Chen, and S. Y. Ho, J. Polym. Sci., Polym. Chem. Ed., 34, 2613 (1996).

10. T. C. Chang, Y. C. Chen, Y. S. Chiu, and S. Y. Ho, Polymer, 37 , 2963 (1996).

11. T. C. Chang, H. B. Chen, S. Y. Ho, and Y. S. Chiu, J. Macromol. Sci. Pure Appl. Chem., A33, 1263 (1996).

12. R. Kuhn and H. Trisch, Monatsh. Chem., 95, 457 (1964).

13. H. B. Chen, T. C. Chang, Y. S. Chiu, and S. Y. Ho, J. Polym. Sci. Poly. Chem. Ed., 33, 679 (1995).

14. K. Ito, J. Polym. Sci., Poly. Chem. Ed., 18, 701 (1980).

15. M. Niwa, T. Matsumoto, and H. Izumi, J. Macromol. Sci. Pure Appl. Chem., A24, 567 (1987).

16. T. Milczarska and J. Szafko, Makromol. Chem., 218, 171 (1994).

17. M. S. Matheson, E. E. Auer, E. B. Bevilacqua, and E. J. Hart, J. Am. Chem. Soc., 73, 1700 (1951).

18. A. Onen, S. Denizligil, and Y. Yagci, Makromol. Chem., 217, 79 (1994). 